\title{
What lies beneath? Herbal medications can lead to adrenal insufficiency
}

Authors: Lauren Quinn ${ }^{1}$, Punith Kempegowda ${ }^{2}$, Lisa Shepherd ${ }^{3}$, Samina Kauser ${ }^{3}$, Briony Johnson ${ }^{3}$, Alexander Lawson ${ }^{3}$, Andrew Bates ${ }^{2}$

${ }^{1}$ University of Hospitals of Leicester

2 Institute of Metabolism and Systems Research, University of Birmingham

3 Department of Endocrinology, Birmingham Heartlands Hospital (BHH)

Correspondence: lauren.Quinn@uhl-tr.nhs.uk

\section{Introduction:}

Complementary therapies form a lifeline for patients when modern medicine cannot meet their expectations.

However, unlike prescription therapy, herbal treatments are not formerly regulated and patients are often not appropriately informed of the benefits and risks of treatment. Furthermore, precise contents of herbal remedies are often incomplete, hence misinforming patients and clinicians alike.

Here, we present the case of a patient in whom we suspect a herbal remedy was responsible for significant morbidity.

\section{Case Presentation: \\ -A 62 year old Asian British female attended the Endocrinology
department with weight gain and fatigue.
- Past medical history included type two diabetes, asthma,
anxiety and depression. \\ -A 62 year old Asian British female attended the Endocrino
department with weight gain and fatigue.
- Past medical history included type two diabetes, asthma,
anxiety and depression. \\ -A 62 year old Asian British female attended the Endocrino
department with weight gain and fatigue.
- Past medical history included type two diabetes, asthma,
anxiety and depression. \\ -A 62 year old Asian British female attended the Endocrino
department with weight gain and fatigue.
- Past medical history included type two diabetes, asthma,
anxiety and depression.

- Drug history revealed she had received multiple courses of prednisolone and regular Clenil Modulite inhaler usage as treatment for exacerbations of asthma in the community, although asthma had never been formerly diagnosed.

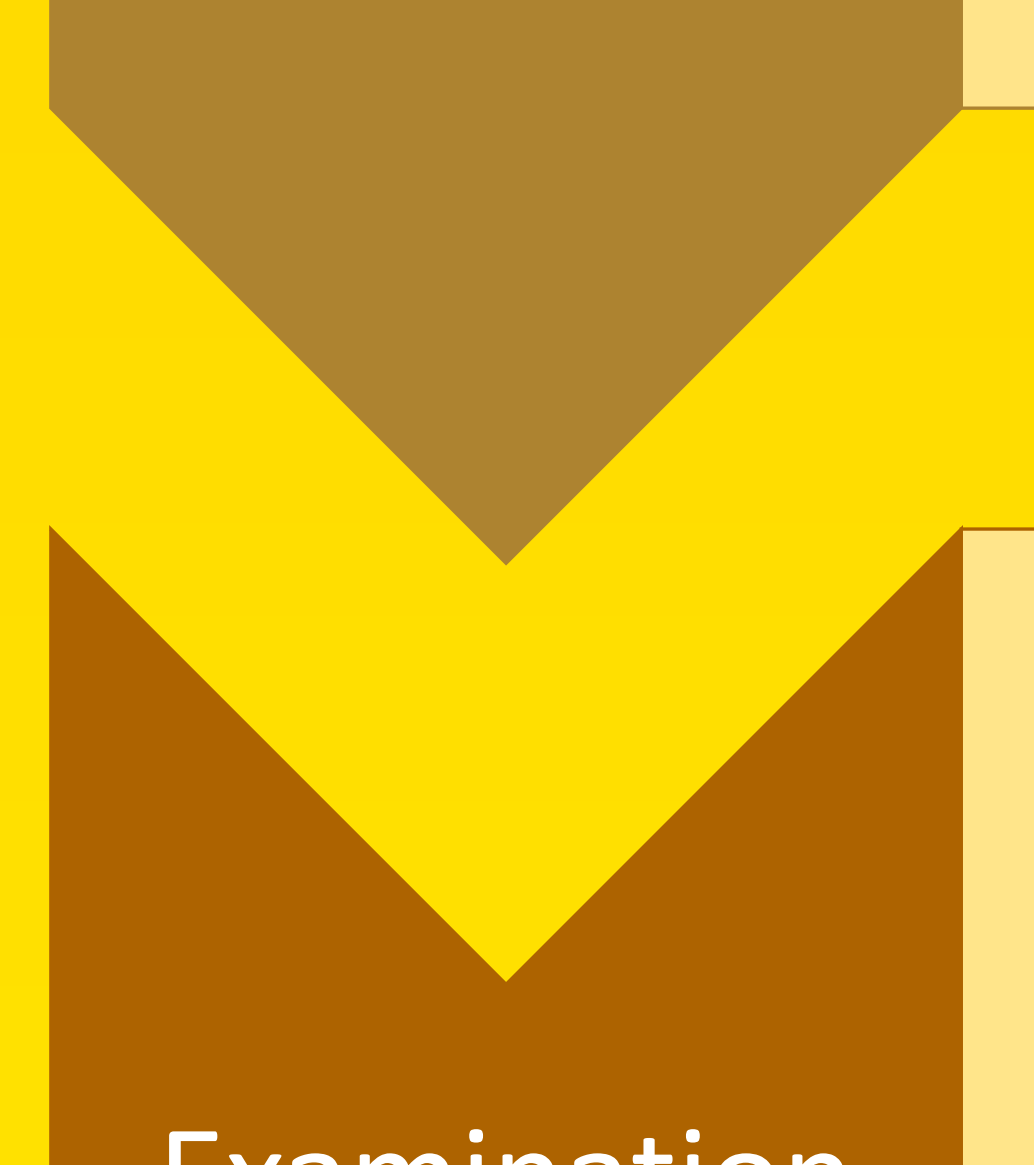

- On examination, there were no signs of abnormal skin pigmentation.

- She was hypertensive. Body Mass Index was $43 \mathrm{~kg} / \mathrm{m}^{2}$. Systemic examination was unremarkable.

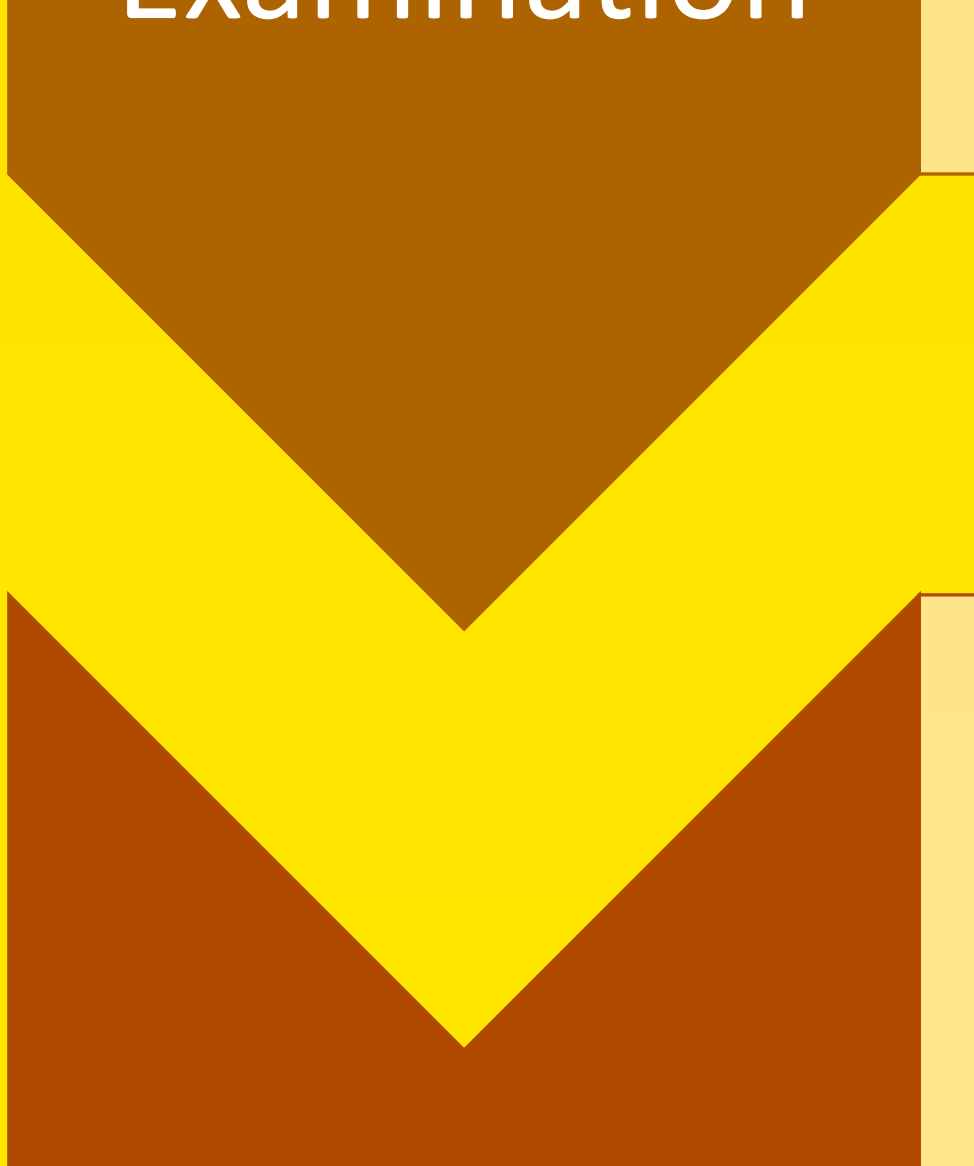

- Cortisol was undetectable at $<28 \mathrm{nmol} / \mathrm{l}$.

- Short synacthen test failed to mount a response.

- Glycosylated Haemoglobin had acutely worsened from 48 to

Investigations

- Following endocrine and respiratory review, the Seretide inhaler and prednisolone were weaned and stopped.

\section{$85 \mathrm{mmol} / \mathrm{mol}$.}

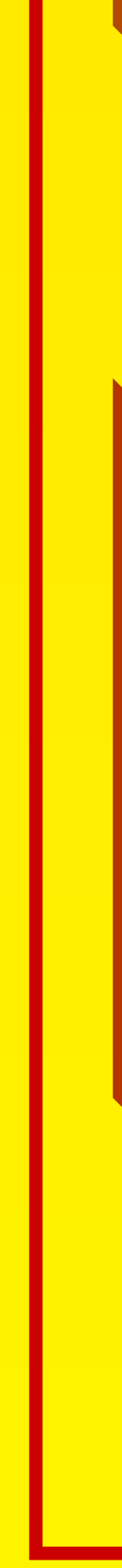

HEART of ENGLAND

\section{University Hospitals Birmingham WHS}

\section{References:}

1. Jonas. The evolution of complementary and alternative medicine (CAM) in the USA over the last 20 years. Forsch Komplementmed. 2013.

2. Chong. Corticosteroid adulteration in proprietary Chinese medicines: a recurring problem. Hong Kong Med J. 2015.

3. Gupta SK. Misuse of corticosteroids in some of the drugs dispensed as preparations from alternative systems of medicine in India. Pharmacoepidemiol Drug Saf. 2000.

\section{Case Outcome:}

Symptoms persisted despite discontinuing prescription steroids. Subsequently, it transpired the patient had been using a non-prescription herbal remedy, for the last ten years.

This herbal remedy was analysed by the BHH Pathology department and found to contain trace elements of:

$\begin{array}{lll}* \text { Dexamethasone } & \text { Diclofenac } \\ \text { * Ciprofloxacin } & \text { Ibuprofen } \\ \text { Paracetamol } & * \text { Cimetidine }\end{array}$

Figure 1a

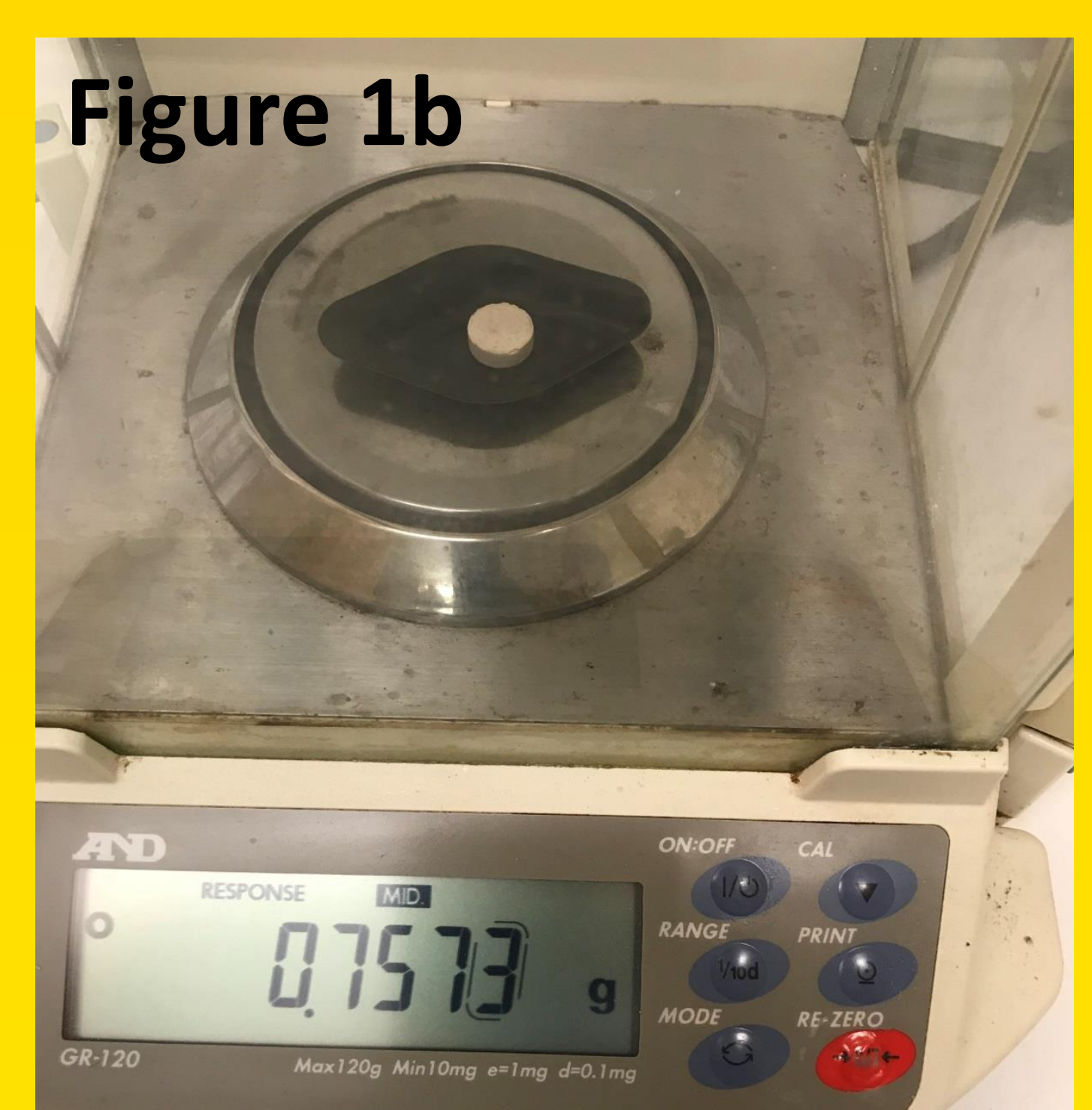

Figures $1 \mathrm{a}$ and $1 \mathrm{~b}$ : Photographs of the Herbal remedy, demonstrating size (in centimetres) and weight (in grams)

\section{Case summary:}

* We deduce that the herbal remedy, containing Dexamethasone, led to secondary adrenal insufficiency, by suppressing the hypothalamic-pituitary axis.

* This likely resulted in deterioration of her glycaemic control, worsening bone mineral density and increased cardiovascular risk given the hypertension and weight gain.

\section{Discussion:}

* Case reports and observational studies have found Chinese and Indian herbal remedies to frequently contain high-dose steroids.

* And if this is demonstrated, patients should be weaned off the steroid agent, whilst started on Hydrocortisone, at least until adrenal function is restored.

\section{Case Learning points:}

1. Herbal remedies frequently contain steroids.

2. This can induce symptomatic and pathological secondary adrenal insufficiency, especially in conjunction with glucocorticoid containing medications.

3. A complete drug history should be ascertained in all patients to help prevent harm arising from secondary adrenal insufficiency. 